\title{
Opportunistic Relaying for Cognitive Radio Enhanced Cellular Networks: Infrastructure and Initial Results
}

\author{
Markus Dominik Mueck ${ }^{\# 1}$, Marco Di Renzo ${ }^{\# 2}$, Mérouane Debbah ${ }^{\# 3}$ \\ ${ }^{\# 1}$ Infineon Technologies AG, IFAG WLS IPA \\ Am Campeon 1-12, 85579 Neubiberg, GERMANY \\ MarkusDominik.Mueck@infineon.com \\ ${ }^{\# 2}$ Laboratory of Signals and Systems (LSS), CNRS - SUPÉLEC - University of Paris-Sud XI \\ 3 rue Joliot-Curie, 91192 Gif-sur-Yvette Cedex, FRANCE \\ marco.direnzo@lss.supelec.fr \\ \#3 Alcatel-Lucent Chair on Flexible Radio, SUPÉLEC \\ 3 rue Joliot-Curie, 91192 Gif-sur-Yvette Cedex, FRANCE \\ marco.direnzo@lss.supelec.fr
}

\begin{abstract}
In this paper, we introduce the application of CRbased spectrum sharing concepts to enable opportunistic relaying operations in cellular networks. In particular, i) we build on recent FCC rules and CEPT plans (SE43) that regulate the opportunistic spectrum usage in TV white spaces at 470-790 MHz; and ii) we show that opportunistic relaying can lead to higher spectral efficiencies and/or reduced CAPEX (and consequently OPEX) due to a lighter infrastructure deployment. Finally, we outline some issues that are important to be addressed to quantify the gains achieved by the proposed opportunistic relay-based infrastructure deployment with respect to existing cellular networks. Some preliminary results are also shown.
\end{abstract}

\section{INTRODUCTION}

Since the original Cognitive Radio (CR) concept had emerged [1], [2], the opportunistic usage of selected frequency bands has become one of the most promising application scenarios from the perspective of a near- to mid-term broad market deployment [3], [4]. Driven by the prospect of a hugely increased overall spectral efficiency through the opportunistic exploitation of otherwise unused frequency bands and the favorable propagation conditions in some of the considered bands (such as the TV white spaces at 470-790 $\mathrm{MHz}$ [5]), a series of large-scale research projects have studied the practical feasibility of such system concepts. Some well-known examples include the European funded projects IST-E2R, IST-E2R II [6], ICT-E3 [7], etc. Moreover, the Federal Communications Commission (FCC) in the USA has defined a set of rules for deploying Cognitive Radio Systems (CRS) [8], and related standardization activities [9] have taken up these results in order to work towards the definition of an effective industrial product. For example, i) the ETSI Reconfigurable Radio System (RRS) [10] works towards CR and Software Defined Radio (SDR) solutions, ii) the IEEE 802.22 working group [11] was created in 2004 to develop a standard for Wireless Regional Area Networks (WRAN) exploiting the white spaces available in the TV frequency spectrum, and iii) the Standards Coordinating Committee 41 (SCC41), i.e., the former IEEE 1900 Standards Committee, is working towards the development of standards related to dynamic spectrum access networks [12]. More recently, a new CR boom set in and several novel groups were created at the end of 2009: i) the IEEE 802.11af working group has been created to introduce some modifications to both the 802.11 Physical (PHY) layer and the 802.11 Medium Access Control (MAC) layer, to meet the legal requirements for channel access and coexistence in the TV white spaces [13]; and ii) the IEEE 802.19 working group, on the other hand, is working towards the development of standards for allowing the coexistence between wireless standards of unlicensed devices [14].

It can be observed, however, that most of the above mentioned standardization activities are mainly related to typical ad hoc networks to be deployed in home scenarios. In this paper, on the other hand, we suggest the application of CR-based spectrum sharing concepts to enable opportunistic relaying operations in cellular networks [15]-[19]. The infrastructure deployment introduced in this paper for cellular networks, which is based on the opportunistic exploitation of white spaces in the TV band, is shown to get the following advantages:

- It increases the spectral efficiency of the communication system, by exploiting the lower propagation losses experienced by the electromagnetic waves propagating in the TV frequency bands at $470-790 \mathrm{MHz}$ with respect to typical cellular frequencies at $2 \mathrm{GHz}$;

- It achieves a higher system throughput for the same relay transmit power;

- It increases the coverage area of a relay node and offers opportunities for macro-diversity gains;

- It reduces the CAPEX (CAPital EXpenditure), and consequently OPEX (OPerating EXpense), for cellular operators by requiring a less dense infrastructure deployment ("lighter deployment").

The reminder of this paper is organized as follows. Section II introduces some relay-assisted infrastructure deployment possibilities to opportunistically exploit the TV white spaces for cellular networks. Section III describes important aspects 
to be investigated and accurately studied in order to quantitatively assess the advantages introduced by the proposed CR-enhanced cellular architecture with respect to conventional cellular-based solutions. Moreover, some preliminary results for a simple three-node network are shown by using the IEEE $802.16 \mathrm{j}$ channel model developed for evaluating the performance of multi-hop systems. Finally, Section IV concludes the paper.
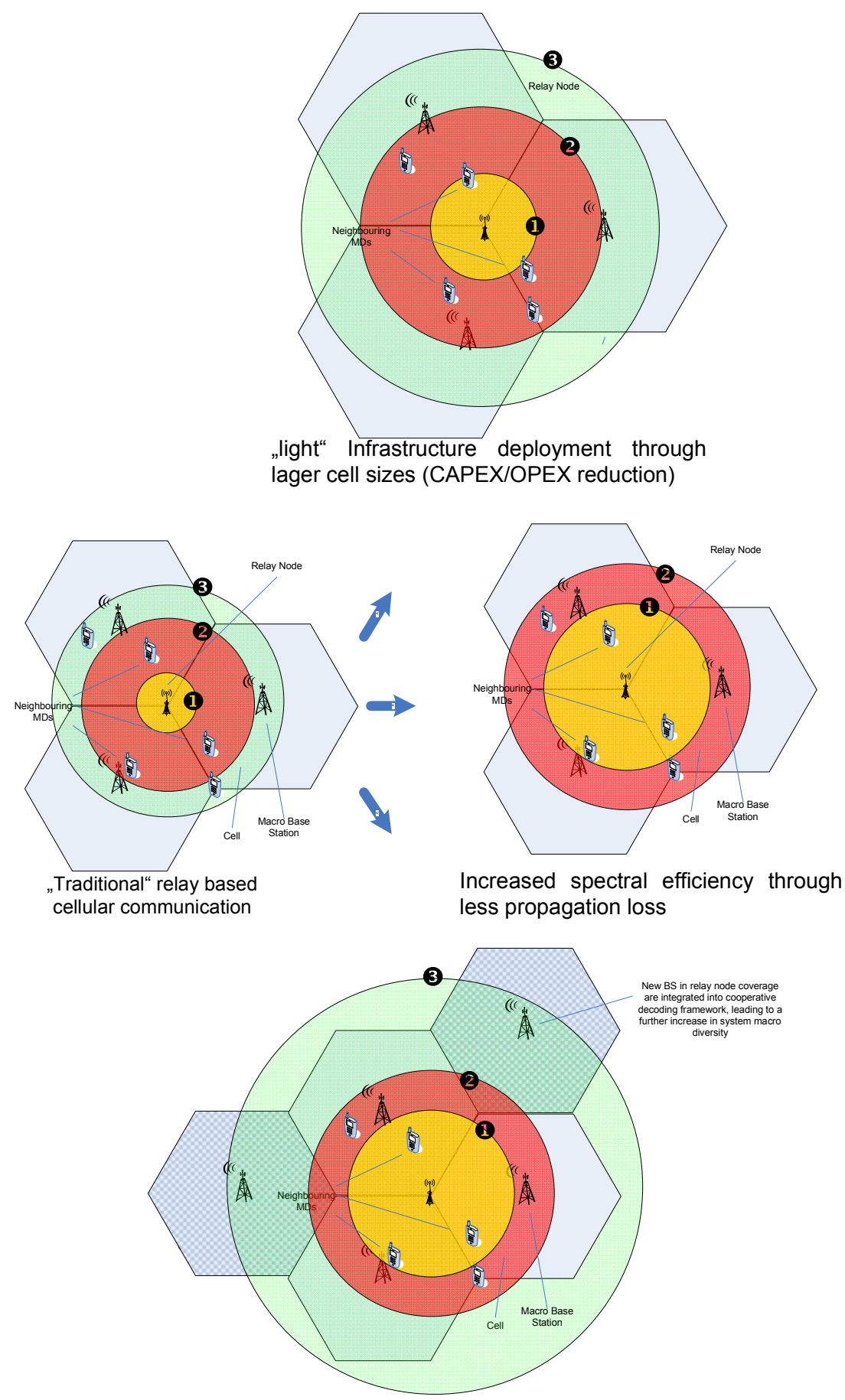

Increased spectral efficiency through extended macro diversity (BS integrated into cooperative decoding of relay-node signals)

Fig. 1: Opportunistic relaying scenarios based on TV white spaces (colour circles around the relay node indicate the area in which the signal for a given link parameterization, i.e., code rate, modulation type, output power level, etc., is correctly decoded). 


\section{OPPORTUNISTIC RELAY-ASSISTED INFRASTRUCTURES FOR CELLULAR NETWORKS}

In this section, we describe the benefits that can be potentially achieved by relay-based cellular networks, which opportunistically exploit the available white spaces in the TV frequency band (470-790 MHz) for relaying operations. The traditional relay-based cellular architecture is shown in the left-hand side of Fig. 1. In particular, Fig. 1 depicts a Mobile Device (MD) which wants to send data to a macro Base Station (BS), and vice versa. To reduce the transmission power, increase the link quality, or improve the system diversity, the MD might opportunistically exploit a relay node, which is willing to forward data to the macro BS of interest. Throughout this paper, we assume that the communications with the relay node (both the MD-to-relay and relay-to-BS, and vice versa) occur over the TV frequency band, when white spaces are available and are correctly detected in this band.

The fundamental advantage of exploiting the white spaces in the TV band for opportunistic relying resides in the reduced propagation losses experienced by the wireless signals in the 470-790 $\mathrm{MHz}$ band, when compared to conventional higher frequency bands used for cellular networks, e.g., the $2 \mathrm{GHz}$ band. This introduces three main potential benefits:

a) Lighter infrastructure deployment through larger cell sizes. If we assume that the probability of a white space in the TV band being available for opportunistic relaying is high, the lower propagation losses experienced by the wireless signals, over the TV band, allow us to increase the cell size of the cellular system, as shown in the upper right-hand side of Fig. 1. As a matter of fact, due to the reduced propagation losses, original spectral efficiencies and average data throughput levels can be maintained even using larger cell sizes. This results in a less dense infrastructure deployment of the macro BS, which, in turn, reduces CAPEX and OPEX of cellular operators. For those scenarios where the probability of available white spaces might not be very high, the required Quality of Service (QoS) might still be guaranteed by applying the most robust communication mode (low channel code rate, robust modulation, high output power levels, etc.) available for the used communication protocol.

b) Increased spectral efficiency through reduced propagation loss. Instead of increasing the cell size while keeping the same spectral efficiency, the reduced propagation losses over the TV band can be exploited to switch the link parameters of the communication protocol to a less robust setting (i.e., channel code rate, modulation type, etc.), thus yielding a higher spectral efficiency and a higher data throughput. This new cellular infrastructure is sketched in the right-hand side of Fig. 1.

c) Increased spectral efficiency through extended macro diversity. Instead of increasing the cell size (see, a)) or modifying the modulation and coding parameters at the physical layer (see, b)), we can consider a third option where the cellular network in Fig. 1 opportunistically exploits the relay node by keeping all link parameters as in a non-relay-based deployment. In such a case, due to the reduced propagation losses over the TV band, the signal emitted by the relay can be overheard by more distant macro BS and exploited in a cooperative setting to get some macro-diversity gains. This allows the system to improve the performance via cooperative diversity protocols. This scenario is shown in the lower right-hand side of Fig. 1.

\section{A. Relaying Schemes}

The system architecture described in Fig. 1 exploits the principle of relay and multi-hop networks for communication [15]. Looking at the literature, there are several protocols that have been proposed for relaying so far (see, e.g., [20]-[22]). Some notable examples of relaying schemes are: i) Amplifyand-Forward (AF), where the relays act as analog repeaters by retransmitting an amplified version of their received signals. They are usually subject to an increased noise floor; ii) Decode-and-Forward (DF), where the relays attempt to decode, regenerate and retransmit an exact copy of the original signal. They are usually subject to error decoding propagation issues; and iii) Decode-and-Re-encode (DR), where the relays attempt to decode and construct codewords that are different from the received codewords, thereby providing incremental redundancy to a receiver that receives the original and the re-encoded signals. Similar to the DF scheme, there can be an error propagation problem in this case as well. A recent survey describing more advanced alternatives can be found in [23].

\section{EVALUATION OF EFFICIENCY GAINS: OPEN ISSUES AND INITIAL RESULTS}

The solutions proposed in Section II can potentially provide several benefits and improve the performance of conventional cellular systems. In particular, a quantitative assessment of advantages and disadvantages of the three scenarios envisaged in Fig. 1 requires a deep understanding of the following open issues:

Accurate evaluation of the larger coverage area allowed by transmitting over the $470-790 \mathrm{MHz}$ frequency band rather than over the $2 \mathrm{GHz}$ frequency band.

- Accurate evaluation of the spectral efficiency gains achievable by using the TV frequency band for typical values of the infrastructure density of BSs (scenario b) in Section II), and for a less dense BS deployment (scenario a) in Section II).

- Accurate evaluation of the spectral efficiency gains achievable by using the TV frequency band by exploiting cooperative diversity protocols among neighboring BSs.

- Accurate evaluation of the inter-cell interference when employing the TV frequency band for typical infrastructure deployment densities operating at $2 \mathrm{GHz}$ (scenario c) in Section II).

- Accurate evaluation of the system performance when the cellular network needs to switch back to the conventional 
setting in Fig. 1, e.g., due to the absence of white spaces in the TV band.

In this paper, we provide some initial results to show the possible performance gains that can be achieved by opportunistically using the TV frequency band for data transmission. In particular, the potential benefits described in Section II arise from the reduced propagation losses experienced by the signals propagating over a low frequency band. The aim of this section is to quantify the performance improvement by using a parametric path-loss model, such as that defined within the IEEE 802.16j standardization activities [24].

\section{A. Reference Scenario}

We consider a simple three-node relay scheme, which is composed by: i) a source (S), ii) a destination (D), and iii) a relay $(\mathrm{R})$, which is opportunistically used to relay the signal from $\mathrm{S}$ to $\mathrm{D}$ in a typical time-orthogonal dual-hop relay fashion. We consider two case-studies:

a) The first one assumes that only the legacy communication channel over a $2 \mathrm{GHz}$ carrier frequency is available for transmission and used for communication in a dual-hop (i.e., S-to-R-to-D) relay fashion.

b) The second one assumes that a spectrum hole is available in the TV frequency band $(470-790 \mathrm{MHz})$ and $\mathrm{S}$ starts transmitting over this frequency band, by still keeping the same dual-hop communication scheme.

With regard to the relaying mechanism, for illustrative purposes, we consider a simple AF method with full channel inversion by the relaying node and time-orthogonal access to the wireless resources [25]. As far as the propagation scenario is considered, we assume a Rayleigh fading channel with the ART-to-ART (type D: Line-of-Sight, LOS) path-loss model in [24] for both wireless links. Finally, for the sake of simplicity, the relay $\mathrm{R}$ is assumed to be located at half distance between the source $\mathrm{S}$ and the destination $\mathrm{D}$.

With the assumptions above and by considering a Differential Binary Phase Shift Keying (D-BPSK) modulation scheme, the end-to-end Average Bit Error Probability (ABEP) at the destination can be written as follows [26, Eq. (21)]:

$$
\mathrm{ABEP}=\frac{1}{2}{ }_{2} F_{1}\left(1,2 ; \frac{3}{2} ;-\frac{\bar{\gamma}}{4}\right)
$$

where ${ }_{2} F_{1}(,, \because, \cdot)$ is the Gaussian hypergeometric function defined in [23, Eq. (15.1.1)], and $\bar{\gamma}$ is the average Signal-toNoise-Ratio (SNR) for each link, which embeds the path-loss model, i.e., $\bar{\gamma}_{[\mathrm{dB}]}=\bar{\gamma}_{[\mathrm{dB}]}(d, f)=\gamma_{[\mathrm{dB}]}^{(0)}-\mathrm{PL}_{[\mathrm{dB}]}(d, f)$, where $\gamma_{[\mathrm{dB}]}^{(0)}$ is the SNR when there are no propagation losses, $\operatorname{PL}_{[\mathrm{dB}]}(\cdot, \cdot)$ is the path-loss in [24], $f$ is the carrier frequency used for transmission, and $d=d_{S R}=d_{R D}$ is the S-to-R and the R-to-D distances.

\section{B. Numerical Results}

In Figs. 2-4, we show some numerical results in order to illustrate the different performance achievable by transmitting over the conventional legacy $2 \mathrm{GHz}$ frequency band and over the TV frequency band by opportunistically using the available white spaces. The curves are shown for different distances $d=d_{S R}=d_{R D}$, for various combinations of heights $\left(h_{S}, h_{R}, h_{D}\right)$ of $\mathrm{S}, \mathrm{R}$, and $\mathrm{D}$ with respect to the ground floor, and for various carrier frequencies that can be opportunistically used in the TV frequency band at $470-790$ $\mathrm{MHz}$.

Overall, the numerical results confirm the potential advantages mentioned in Section II, and, in particular, the reduced path-loss experienced over the TV frequency band can be exploited in three different ways: i) by keeping the same modulation and transmitting less power to get the same ABEP (green solution), ii) by switching the modulation scheme (to an higher order) for increasing the spectralefficiency and keeping the same ABEP, and iii) by keeping the same parameters and getting a better ABEP. For example, in Fig. 2 we observe that for $h_{S}=h_{R}=h_{d}=6.5 \mathrm{~m}$ we could transmit over a wireless link at $470 \mathrm{MHz}$ with $d=200 \mathrm{~m}$ by getting the same performance as a legacy wireless link at 2 $\mathrm{GHz}$ with $d=100 \mathrm{~m}$. In this setup, the communication distance can be increased twofold by keeping the same QoS/ABEP.

\section{CONCLUSION}

In this paper, we have introduced the application of CRbased spectrum sharing concepts to enable opportunistic relaying operations in cellular wireless networks. The potential benefits of exploiting, in an opportunistic fashion, the TV frequency band for relaying have been outlined and possible modifications for a cellular-like system architecture have been discussed. Finally, some numerical results, for a simplified reference scenario, have been shown and the performance gain due to the reduced propagation losses over the TV frequency band have been quantified by using the IEEE 802.16j path-loss model.

\section{ACKNOWLEDGMENT}

This work was supported by the Alcatel-Lucent Chair on Flexible Radio.

\section{REFERENCES}

[1] J. Mitola III, "Cognitive Radio", Ph.D. thesis, KTH, Stockholm, Sweden, 2000.

[2] I. F. Akyildiz, W.-Y. Lee, M. C. Vuran, and S. Mohanty, "NeXt generation/dynamic spectrum access/cognitive radio wireless networks: A survey", Computer Networks, vol. 50, pp. 2127-2159, May 2006.

[3] A. Shukla et al., "Cognitive radio technology. A study for Ofcom - Vol. 1", Final Report, QINETIQ/06/00420 Issue 1.1, Feb. 2007.

[4] "Choice, competition, innovation, delivering the benefits of the digital dividend", UK Office of Commun., Dec. 2007.

[5] E. Visotsky, S. Kuffner, and R. Peterson, "On collaborative detection of TV transmissions in support of dynamic spectrum sensing", IEEE Symp. New Frontiers in Dynamic Spectrum Access Networks, pp. 338345 Nov. 2005. 
[6] D. Bourse, M. Muck, D. Bateman, S. Buljore, N. Alonistioti, K Moessner, E. Nicollet, E. Buracchini, P. Demestichas, M. Stamatelatos, and E. Patouni, "FP6 E2R Programme Achievements and Impact", proc. of SDR 07 Technical Conference and Product Exposition. [Online].

Available: http://www.sdrforum.org/pages/sdr07/Proceedings/Papers/Invited/12.5001 invitedPaper1 Bourse.pdf.

[7] ICT-2007-216248 E3 Project, http://www.ict-e3.eu.

[8] http://www.fcc.gov/oet/cognitiveradio.

[9] M. Sherman, A. N. Mody, R. Martinez, C. Rodriguez, and R. Reddy, "IEEE standards supporting cognitive radio and networks, dynamic spectrum access, and coexistence", IEEE Commun. Mag., pp. 72-79, July 2008.

[10] ETSI Reconfigurable Radio Systems

(RRS), http://www.etsi.org/WebSite/technologies/RRS.aspx.

[11] IEEE 802.22, Working Group on Wireless Regional Area Networks ("WRANs"), http://www.ieee802.org/22.

[12] http://www.scc41.org/.

[13] [13] IEEE 802.11 Working Group, Task Group af, http://grouper.ieee.org/groups/802/11/Reports/tgaf update.htm.

[14] IEEE 802.19 Wireless Coexistence Working Group, http://www.ieee802.org/19.

[15] R. Pabst, B. H. Walke, D. C. Schultz, P. Herhold, H. Yanikomeroglu, S. Mukherjeee, H. Viswanathan, M. Lott, W. Zirwas, M. Dohler, H. Aghvami, D. D. Falconer, and G. P. Fettweis, "Relay-based deployment concepts for wireless and mobile broadband radio", IEEE Commun. Mag., vol. 42, no. 9, pp. 80-89, Sep. 2004.

[16] M. Dohler, S. A. Ghorashi, M. Ghozzi, M. Arndt, F. Said, and A. H. Aghvami, "Opportunistic scheduling using cognitive radio", Comptes Rendus Physique: Towards Reconfigurable and Cognitive Communications, vol. 7, no. 7, pp. 805-815, Sept. 2006.
[17] O. Oyman, "Opportunistic scheduling and spectrum reuse in relaybased cellular OFDMA networks", IEEE Global Telecommun. Conf., pp. 3699-3703, Nov. 2007.

[18] O. Oyman, J. N. Laneman, and S. Sandhu, "Multihop Relaying for Broadband Wireless Mesh Networks: From Theory to Practice", IEEE Commun. Mag., vol. 45, no. 11, pp. 116-122, Nov. 2007.

[19] Y. Guo, G. Kang, N. Zhang, W. Zhou, and P. Zhang, "Outage Performance of relay-assisted cognitive-radio system under spectrumsharing constraints", IEEE Electron. Lett., vol. 46, no. 2, pp. 182-184, Feb. 2010.

[20] J. Laneman, D. Tse, and G. Wornell, "Cooperative diversity in wireless networks: efficient protocols and outage behavior", IEEE Trans. Inform. Theory, vol. 50, no. 12, pp. 3062-3080, Dec. 2004.

[21] J. Laneman and G. Wornell, "Distributed space-time-coded protocols for exploiting cooperative diversity in wireless networks", IEEE Trans. Inform. Theory, vol. 49, no. 10, pp. 2415-2425, Oct. 2003.

[22] P. Herhold, E. Zimmermann, and G. Fettweis, "Cooperative multi-hop transmission in wireless networks", Computer Networks, vol. 49, no. 3 , pp. 299-324, Oct. 2005

[23] M. Uysal, Cooperative Communications for Improved Wireless Network Transmission: Framework for Virtual Antenna Array Applications, Information Science Reference, July 2009.

[24] http://www.ieee802.org/16/relay/docs/80216j-06 013r3.pdf.

[25] M. Di Renzo, F. Graziosi, and F. Santucci, "A unified framework for performance analysis of CSI-assisted cooperative communications over fading channels", IEEE Trans. Commun., vol. 57, no. 9, pp. 2552-2557, Sept. 2009.

[26] M. O. Hasna and M.-S. Alouini, "End-to-end performance of transmission systems with relays over Rayleigh-fading channels", IEEE Trans. Wireless Commun., vol. 2, pp. 1126-1131, Nov. 2003.

[27] M. Abramowitz and I. A. Stegun, Handbook of Mathematica Functions With Formulas, Graphs, and Mathematical Tables, 9th ed. New York: Dover, 1970.
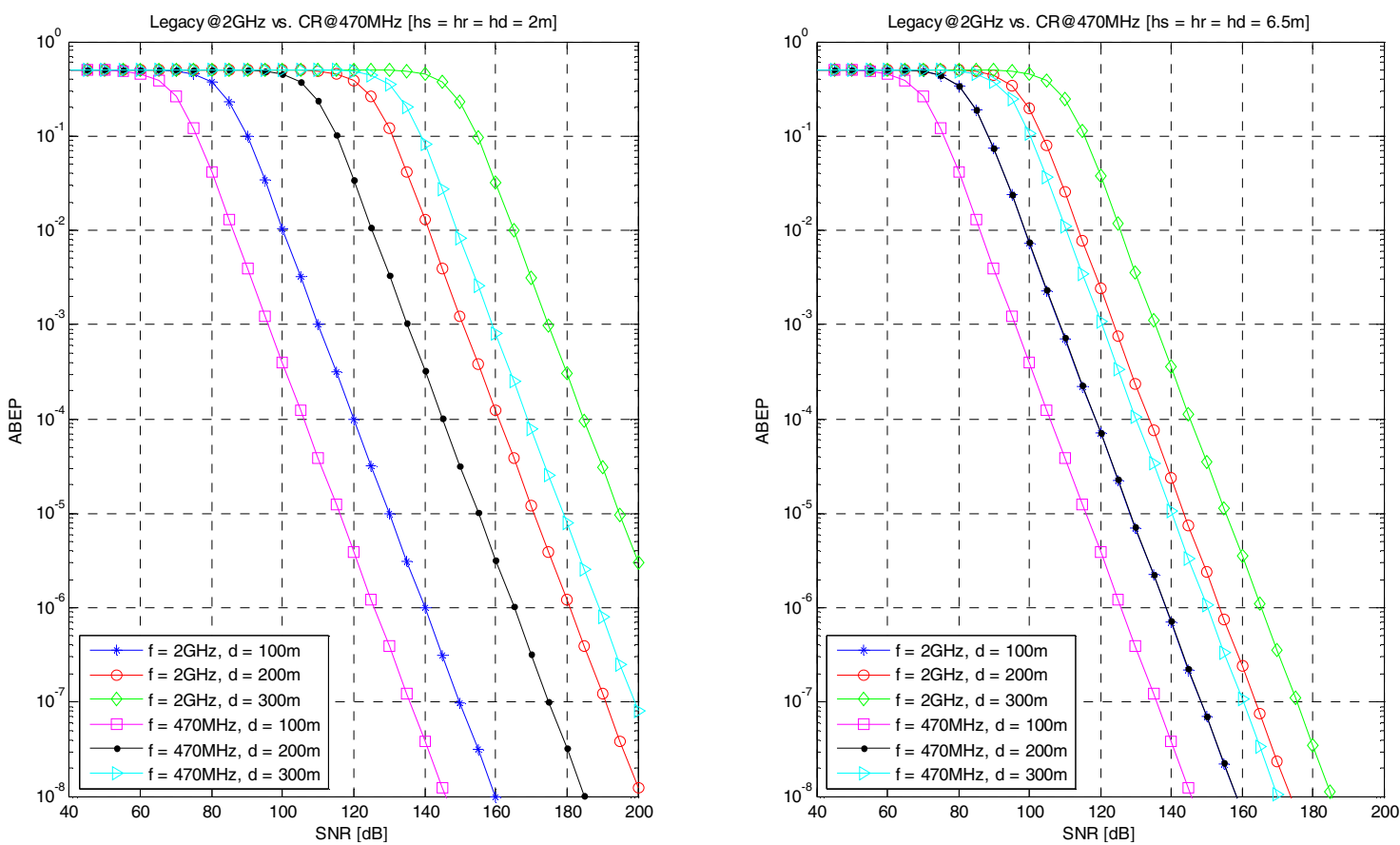

Fig. 2: ABEP over a TV frequency band at $470 \mathrm{MHz}\left(\mathrm{SNR}[\mathrm{dB}]=\gamma_{[\mathrm{dB}]}^{(0)}\right)$. 

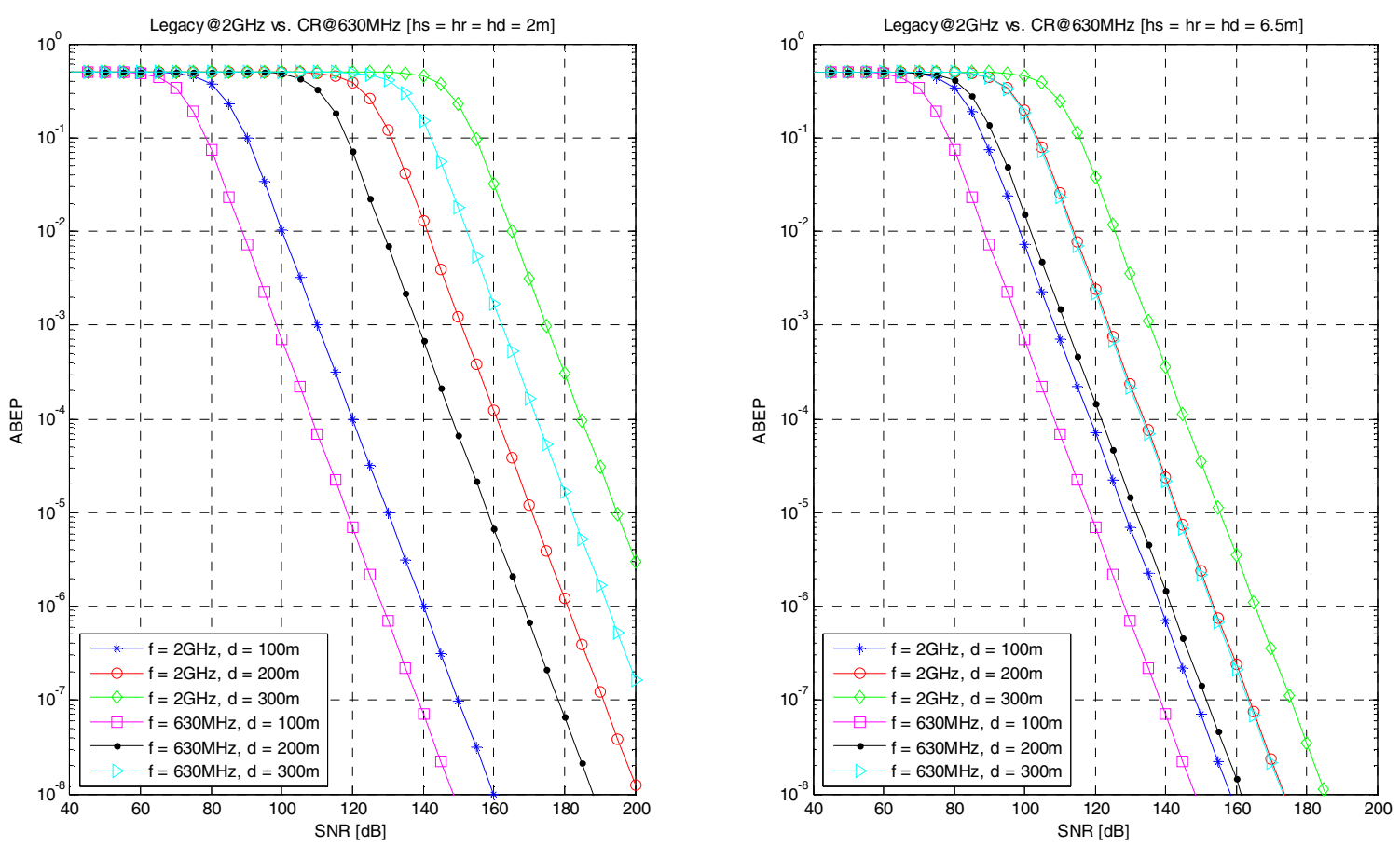

Fig. 3: ABEP over a TV frequency band at $630 \mathrm{MHz}\left(\mathrm{SNR}[\mathrm{dB}]=\gamma_{[\mathrm{dB}]}^{(0)}\right)$.
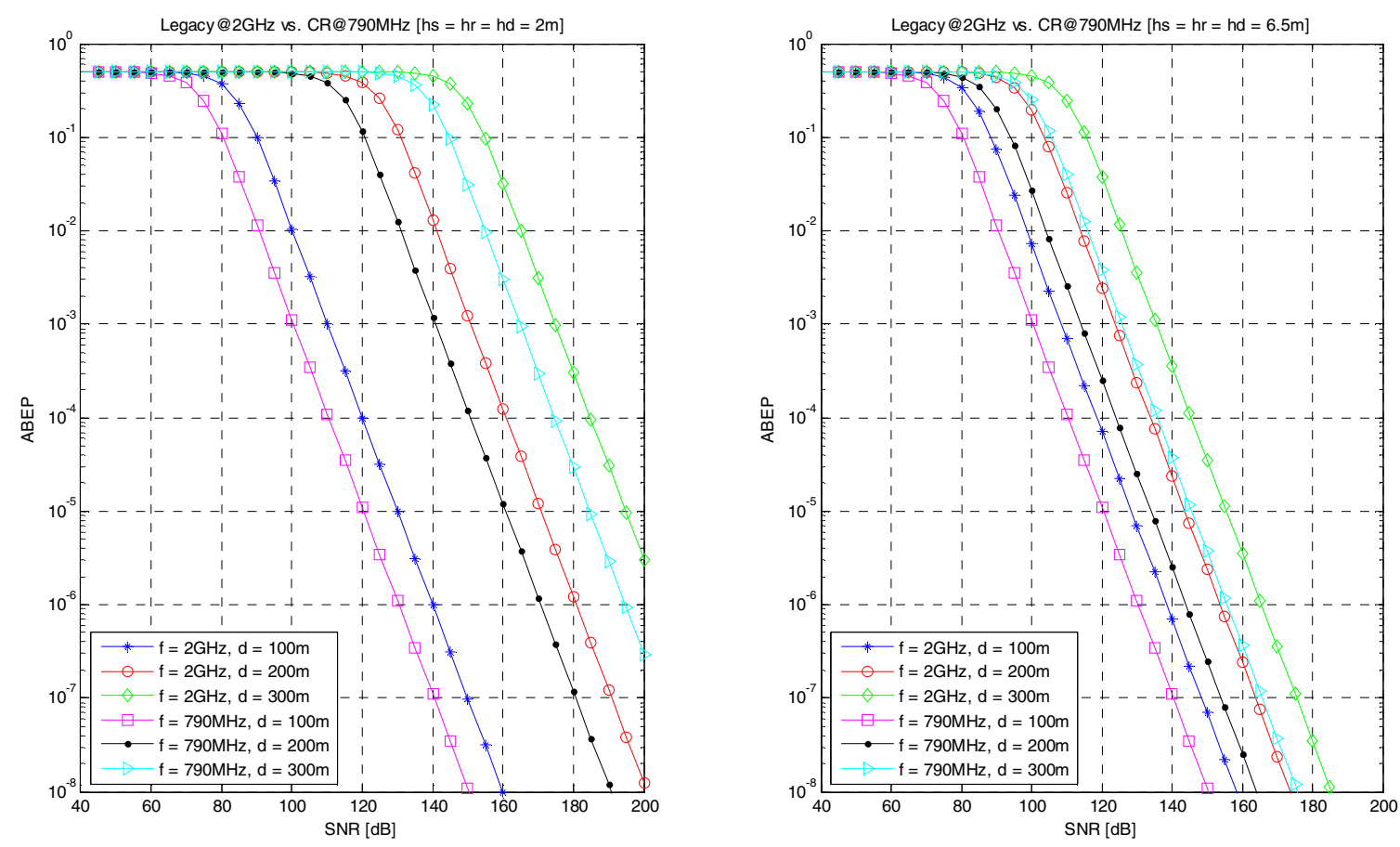

Fig. 4: ABEP over a TV frequency band at $790 \mathrm{MHz}\left(\mathrm{SNR}[\mathrm{dB}]=\gamma_{[\mathrm{dB}]}^{(0)}\right)$. 